EPJ Web of Conferences 61, 01009 (2013)

DOI: $10.1051 /$ epjconf/ 20136101009

(C) Owned by the authors, published by EDP Sciences, 2013

\title{
Energy densities of magnetic field and relativistic electrons at the innermost region of the M87 jet
}

\author{
M. Kino ${ }^{1, a}$, F. Takahara ${ }^{2}$, K. Hada ${ }^{3,4}$, and A. Doi ${ }^{1}$ \\ ${ }^{1}$ ISAS/JAXA, 3-1-1 Yoshinodai, Chuo, Sagamihara 252-5210, Japan \\ ${ }^{2}$ Department of Earth and Space Science, Osaka University, Toyonaka 560-0043, Japan \\ ${ }^{3}$ INAF/IRA, via Gobetti 101, I-40129 Bologna, Italy \\ ${ }^{4}$ NAOJ, Osawa, Mitaka, Tokyo 181-8588, Japan
}

\begin{abstract}
We explore energy densities of magnetic fields and relativistic electrons in M87 jet. Since the radio core at the base of the M87 jet is the optically thick surface against synchrotron self absorption (SSA), observations directly give the size and turnover frequency for SSA. Using the observed angular diameter 0.11 mas, which corresponds to 16 Schwarzschild radii of the central black hole with $6 \times 10^{9}$ solar mass, and the flux density of the radio core at $43 \mathrm{GHz}$, we estimate the energy densities of magnetic field $\left(U_{B}\right)$ and relativistic electrons $\left(U_{e}\right)$ by comparing the standard SSA formula to the observed radio core. Together with the allowed total kinetic power of the M87 jet, we find that (i) the allowed $B$ is limited in the range $2 \mathrm{G} \leq B \leq 13 \mathrm{G}$, and that (ii) $0.18 \leq U_{e} / U_{B} \leq 66$ holds. Our results significantly constrain formation mechanism of relativistic jets in active galactic nuclei.
\end{abstract}

\section{Introduction}

Formation mechanism of relativistic jets in active galactic nuclei (AGNs) remains as a longstanding unresolved problem in astrophysics. Although an importance of estimations of magnetic field energy density $\left(U_{\mathrm{B}}\right)$ and electron one $\left(U_{e}\right)$ for resolving the formation mechanism had been emphasized (e.g., [13]; [5]), it is not observationally clear whether $U_{\mathrm{B}}$ or $U_{e}$ is dominant at a jet base. Deviation from equi-partition (i.e., $U_{e} / U_{B} \approx 1$ ) is essential for investigation of relativistic jet formation. However, no one has succeeded in firmly obtaining a robust estimation of an actual value of $U_{e} / U_{B}$ at a jet base.

M87, a nearby giant radio galaxy located at a distance of $D=16.7 \mathrm{Mpc}$, hosts one of the most massive super massive black hole $M_{\bullet}=(3-6) \times 10^{9} M_{\odot}$ and thus M87 is the best source for investigating a jet base. Furthermore, M87 has been well studied at wavelength from radio to Very High Energy (VHE) $\gamma$-ray ([1] and reference therein) and causality arguments based on VHE $\gamma$ ray outburst in February 2008 indicate that the VHE emission region is less than $\sim 5 \delta \mathrm{R}_{\mathrm{s}}$ where $\delta$ is the relativistic Doppler factor ([2]). VLBA beam resolution at $43 \mathrm{GHz}$ typically attains about $0.21 \times 0.43$ mas which is equivalent to $5.3 \times 10^{16} \times 1.1 \times 10^{17} \mathrm{~cm}$. If we take $M_{\bullet}=6 \times 10^{9} M_{\odot}$, then VLBA beam resolution corresponds to $30 \times 60 \mathrm{R}_{\mathrm{s}}$. Recent progress of VLBI observations reveals the innermost structure of the M87 jet, i.e., frequency and core-size relation, distance and core-size relation down to close to 16 Schwarzschild radii $\left(R_{\mathrm{s}}\right)$ ([12], hereafter H11). Thus,

\footnotetext{
ae-mail: kino@vsop.isas.jaxa.jp
}

the jet base of M87 is the best laboratory for investigation of $U_{e} / U_{B}$ in a real vicinity of a central engine.

Two significant forward steps are recently obtained in M87 observations and the present work is motivated by them. (1) H11 succeed in directly measuring core-shift phenomena at the jet base of M87 at 2, 5, 8, 15, 22 and $43 \mathrm{GHz}$. The radio core position at each frequency has been obtained by the astrometric observation of the core shift along the M87 jet. Since the radio core surface corresponds to the optically-thick surface at each frequency, a synchrotron-self-absorption (SSA) turnover frequency $v_{\text {ssa }}$ is identical to the observed frequency itself. (2) We recently measure core sizes in [10] (hereafter H13). Hereafter we focus on the radio core at $43 \mathrm{GHz}$. In $\mathrm{H} 13$, we select VLBA data observed after 2009 with sufficiently good qualities (all 10 stations participated and good uvcoverages). To measure the width of the core, a single, full-width-half-maximum (FWHM) Gaussian is fitted for observed core in the perpendicular direction to the jet axis and we derive the width of the core $\left(\theta_{\text {FWHM }}\right)$. We stress that the core width is free from the uncertainty of viewing angle. Therefore, using $\theta_{\mathrm{FWHM}}$ at $43 \mathrm{GHz}$, we can estimate model-independent value of $U_{e} / U_{B}$ in the $43 \mathrm{GHz}$ core of M87 for the first time.

\section{One-zone Model}

Here, we derive the explicit expression of $U_{e} / U_{B}$ by using the fundamental formula of SSA process ([9], hereafter GS65; [4], hereafter BG70; [21], hereafter RL79). Two 
physical quantities, $B$ and $K_{e}$ are determined by the comparison of physical quantities measured by VLBA observations (i.e., $\theta_{\mathrm{obs}}, v_{\mathrm{ssa}, \mathrm{obs}}$, and $S_{v_{\mathrm{ssa}}, \mathrm{obs}}$ ) and standard SSA process. In this work, we define the radio spectral index $\alpha$ as $S_{v} \propto v^{-\alpha}$.

Following assumptions are adopted in this work:

- We assume uniform and isotropic distribution of relativistic electrons in the radio core at $43 \mathrm{GHz}$ (the hatched circle in Fig. 1). For M87, polarized flux seems not very large. Therefore, we assume isotropic tangled magnetic field in this work. Hereafter, we denote $B$ as the magnetic field strength perpendicular to the direction of electron motion. Then, the total field strength is given by $B_{\text {tot }}=\sqrt{3} B$.

- We assume the emission region is spherical with its radius $R$ measured at the comoving frame. The radius is defined as $2 R=\theta_{\text {obs }} D$ where $D$ is the distance to a source. There might be a slight difference between $\theta_{\text {FWHM }}$ and $\theta_{\text {obs }}$. VLBI measured $\theta_{\text {FWHM }}$ is conventionally treated as $\theta_{\text {obs }}=\theta_{\text {FWHM }}$, while [17] (hereafter M83) pointed out a deviation expressed as $\theta_{\text {obs }} \approx 1.8 \theta_{\text {FWHM }}$ which is caused by a forcible fitting of Gaussians to a non-Gaussian component. In this work, we introduce a factor $A$ defined as $\theta_{\mathrm{obs}} \equiv A \theta_{\mathrm{FWHM}}$ and $1 \leq A \leq 1.8$ is assumed.

At the radio core, (1) $\tau_{\mathrm{ssa}}=1$ at $v=v_{\mathrm{ssa}}$ holds:

$$
\tau_{\nu_{\mathrm{ssa}}}=2 \alpha_{\nu_{\mathrm{ssa}}} R=1
$$

where $\tau_{v_{\mathrm{sa}}}$, and $\alpha_{v_{\mathrm{ssa}}}$ are the optical depth for SSA, and the absorption coefficient for SSA, respectively. The optically thin emission equals the optically thick one at $v_{\mathrm{ssa}}$ :

$$
\frac{4 \pi}{3} R^{3} \epsilon_{v_{\mathrm{ssa}}}=4 \pi R^{2} S_{v_{\mathrm{ssa}}},
$$

where $\epsilon_{v_{\mathrm{sa}}}$, and $S_{v_{\mathrm{ssa}}}$ are the emissivity and flux per unit frequency, respectively. Via these two relations, we can solve $B$ and $K_{e}$.

The term $K_{e}$, the normalization factor of electron number density distribution $n_{e}(\gamma)$, is defined as

$$
n_{e}\left(\gamma_{e}\right) d \gamma_{e}=K_{e} \gamma_{e}^{-p} d \gamma_{e} \quad\left(\gamma_{e, \min } \leq \gamma_{e} \leq \gamma_{e, \max }\right),
$$

where $p=2 \alpha+1, \gamma_{e, \min }$, and $\gamma_{e, \max }$ are a spectral index, a minimum Lorentz factor, and a maximum Lorentz factor of relativistic electrons, respectively. Let us further review optically thin synchrotron emissions. The maximum in the spectrum of synchrotron radiation from an electron occurs at the frequency: (Eq. 2.23 in GS65)

$$
v_{\mathrm{syn}}=1.2 \times 10^{6} B \gamma_{e}^{2}
$$

where $B$ is the component of magnetic field perpendicular to the direction of the electron motion.

\section{Results}

Obtained expressions for $B$ and $K_{e}$ are as follows;

$$
\begin{aligned}
B & =b(p)\left(\frac{v_{\mathrm{ssa}, \mathrm{obs}}}{1 \mathrm{GHz}}\right)^{5}\left(\frac{\theta_{\mathrm{obs}}}{1 \mathrm{mas}}\right)^{4}\left(\frac{S_{v_{\mathrm{ssa}}, \mathrm{obs}}}{1 \mathrm{Jy}}\right)^{-2} \\
& \times\left(\frac{\delta}{1+z}\right)
\end{aligned}
$$

and

$$
\begin{aligned}
K_{e} & =k(p)\left(\frac{v_{\mathrm{ssa}, \mathrm{obs}}}{1 \mathrm{GHz}}\right)^{-2 p-3}\left(\frac{D}{1 \mathrm{Gpc}}\right)^{-1}\left(\frac{\theta_{\mathrm{obs}}}{1 \mathrm{mas}}\right)^{-2 p-5} \\
& \times\left(\frac{S_{v_{\mathrm{ssa}}, \mathrm{obs}}}{1 \mathrm{Jy}}\right)^{p+2}\left(\frac{\delta}{1+z}\right)^{-p-3}
\end{aligned}
$$

where the numerical coefficients $b(p)$ and $k(p)$ are shown in M83 and [15]. From the above expressions, we can obtain the ratio $U_{e} / U_{\mathrm{B}}$ explicitly as

$$
\begin{aligned}
\frac{U_{e}}{U_{\mathrm{B}}}= & \left(\frac{B_{\mathrm{tot}}^{2}}{8 \pi}\right)^{-1} \int_{\gamma_{e, \min }}^{\gamma_{e, \text { max }}} \gamma_{e} m_{e} c^{2} n_{e}\left(\gamma_{e}\right) d \gamma_{e} \\
= & \frac{8 \pi m_{e} c^{2}}{3(p-2)} \frac{k(p) \gamma_{\min }^{-p+2}}{b^{2}(p)}\left(\frac{\nu_{\mathrm{ssa}, \mathrm{obs}}}{1 \mathrm{GHz}}\right)^{-2 p-13}\left(\frac{D}{1 \mathrm{Gpc}}\right)^{-1} \\
\times & \left(\frac{\theta_{\mathrm{obs}}}{1 \mathrm{mas}}\right)^{-2 p-13}\left(\frac{S_{\nu_{\mathrm{ssa}}, \mathrm{obs}}}{1 \mathrm{Jy}}\right)^{p+6}\left(\frac{\delta}{1+z}\right)^{-p-5} \\
& (\text { for } p>2) .
\end{aligned}
$$

From this, we can estimate $U_{e} / U_{\mathrm{B}}$ without minimum energy (equipartition $B$ field) assumption. It is clear that the measurement of $\theta_{\mathrm{obs}}$ is crucial for determining $U_{e} / U_{\mathrm{B}}$. We argue details on it in the next subsection. It is also evident that a careful treatment of $\gamma_{e, \text { min }}$ is crucial for determining $U_{e} / U_{\mathrm{B}}([14])$.

To evaluate realistic $U_{e} / U_{\mathrm{B}}$, we should take realistic parameter ranges. To this end, we take into uncertainty into account.

Regarding $\alpha$, a simultaneous observation of the spectrum measurement at sub-mm wavelength range is crucial, since most of the observed fluxes at sub-mm range come from the innermost part of the jet. It has been indeed measured by [8] with multi-frequency ALMA observation (cycle 0 ) and it is robust that $\alpha>0.5$ at $>200 \mathrm{GHz}$ where synchrotron emission becomes optically-thin against SSA.

As for allowed $\theta_{\mathrm{obs}}$, we set

$$
0.11 \text { mas } \leq \theta_{\text {obs }} \leq 0.20 \text { mas, }
$$

where we use the average value $\theta_{\mathrm{FWHM}}=0.11$ mas from $\mathrm{H} 13$ and maximum of $\theta_{\mathrm{obs}}$ is 0.11 mas $\times 1.8=0.198$ mas.

The maximal value of $\gamma_{e \text {,min }}$ can be estimated with $\theta_{\mathrm{obs}}=0.20$ mas. Allowed ranges of $\gamma_{e, \min }$ is given by $1 \leq \gamma_{e \text {,min }} \leq 2 \times 10^{2}$, here we set maximum value of $\gamma_{e \text {,min }}$ by requiring synchrotron emission is produced at least above $43 \mathrm{GHz}$. We further impose a condition that timeaveraged bulk kinetic power as inferred from its largescale radio and X-ray morphology $L_{\text {jet }}$ should be smaller than the power at the $43 \mathrm{GHz}$ core

$$
\begin{aligned}
L_{\text {jet }} & \geq \max \left[L_{\text {poy }}, L_{\mathrm{e}}\right], \\
L_{\mathrm{e}} & =\frac{4 \pi}{3} \Gamma^{2} \beta R^{2} c U_{e} \\
L_{\text {poy }} & =\frac{4 \pi}{3} \Gamma^{2} \beta R^{2} c U_{B},
\end{aligned}
$$

where $L_{\text {jet }}$ at large-scale is estimated to be a few $\times 10^{44}$ ergs $^{-1}$ (e.g., [20]). Hereafter, we assume $\Gamma \beta=1$ for simplicity and a slight deviation from this does not influence the main results in this work. Regarding $L_{\mathrm{jet}}$ in the 
M87 jet, we set $5 \times 10^{44} \mathrm{ergs}^{-1} \leq L_{\text {jet }} \leq 3 \times 10^{45} \mathrm{ergs}^{-1}$ where upper limit of $L_{\text {jet }}$ includes an uncertainty due to the deviation from time-averaged $L_{\text {jet }}$ at while flaring phenomena at the jet base may temporally increase $L_{\text {jet }}$ Xray light curve at the M87 core over 10 years showed a flux variation by a factor of $\sim 6$ except for exceptionally high X-ray flux during giant VHE flares which happened in 2008 and 2010 (Fig. 1 in [1]). Based on it, we allow the largest jet kinetic power case as $L_{\text {jet }}=6 \times 5 \times 10^{44} \mathrm{ergs}^{-1}=$ $3 \times 10^{45} \mathrm{ergs}^{-1}$. In this work, we demonstrate the case of $L_{\text {jet }}=3 \times 10^{45} \mathrm{ergs}^{-1}$.

In Fig. 2, we show the allowed $\log \left(U_{e} / U_{B}\right)$ as a function of $\gamma_{e, \text { min }}$ and $B$ with $L_{\text {jet }}=3 \times 10^{45} \mathrm{erg} \mathrm{s}^{-1}$ and $p=3.5$. Note that the field strength $B$ has one-to-one correspondence to $\theta_{\text {obs }}$. From Fig. 2, one can see that the absolute value of $B$ (equivalent to $\theta_{\mathrm{obs}}$ ) is more dominant factor than $\gamma_{e, \text { min }}$ in this allowed ranges. We find that energy density of relativistic electrons dominates over the one of magnetic field $\left(\log \left(U_{e} / U_{B}\right)>1\right)$ when $B<5.7 \mathrm{G}$ and vice versa. The larger $\gamma_{e, \min }$ leads to smaller $\log \left(U_{e} / U_{B}\right)$ because $U_{e}$ becomes smaller for larger $\gamma_{e \text {, min }}$. The allowed $\gamma_{e, \min }$ is limited in $30 \leq \gamma_{e \text {, min }} \leq 2 \times 10^{2}$. The smallest $U_{e} / U_{B}$ realizes at $B \approx 8.1 \mathrm{G}$ and it is $U_{e} / U_{B}=0.18$. The allowed $U_{e} / U_{B}$ is governed by the limit of $L_{\mathrm{j}} \geq \max \left[L_{p o y}, L_{e}\right]$. The lower part of $\log \left(U_{e} / U_{B}\right)$ is determined by the condition of $L_{e}=L_{\mathrm{j}}$, while the upper part is settled by $L_{p o y}=L_{\mathrm{j}}$. The largest $U_{e} / U_{B}$ is realized when $B=2.5 \mathrm{G}$ which shows $U_{e} / U_{\mathrm{B}}=66$.

\section{Summary and discussions}

Based on VLBA observation data at $43 \mathrm{GHz}$, we explore $U_{e} / U_{\mathrm{B}}$ at the base of the M87 jet. We use standard basic theory of synchrotron radiation with the simplest geometry of one-zone sphere model for the radio core at $43 \mathrm{GHz}$. We impose the upper limit of total jet power $L_{\text {jet }}$ based on various previous works. We then find the following things;

- We obtain the allowed range of $B$ as $2 \mathrm{G}<B<13 \mathrm{G}$ in the observed radio core at $43 \mathrm{GHz}$ with its diameter 0.11 mas $\left(\left(16 R_{\mathrm{s}}\right)\right)$. Our estimate of $B$ is basically close to the previous estimate in the literature (e.g., [19]), although fewer assumptions have been made in this work.

Our result excludes a strong magnetic field such as $B \sim$ $10^{3-4} \mathrm{G}$ which is frequently assumed in previous works in order to activate Blandford-Znajek process ([3]). Although M87 has been a prime target for testing relativistic MHD jet simulation studies powered by black-hole spin energy, our result provides a very stringent limit on the maximum $B$,one of the critical parameters in relativistic MHD jets model.

- We obtain the allowed region of $U_{e} / U_{B}$ in the allowed $\theta_{\text {obs }}$ and $\gamma_{e \text {,min }}$ plane. The resultant $U_{e} / U_{B}$ contains both the region of $U_{e} / U_{B}>1$ and $U_{e} / U_{B}<1$. What we should emphasize here is that the deviation from $U_{e} / U_{B} \approx 1$ is not very large. It is found that the allowed range is $0.18 \leq U_{e} / U_{\mathrm{B}} \leq 66$.

This result gives a tight constraint against relativistic MHD models since they seem to postulate much larger

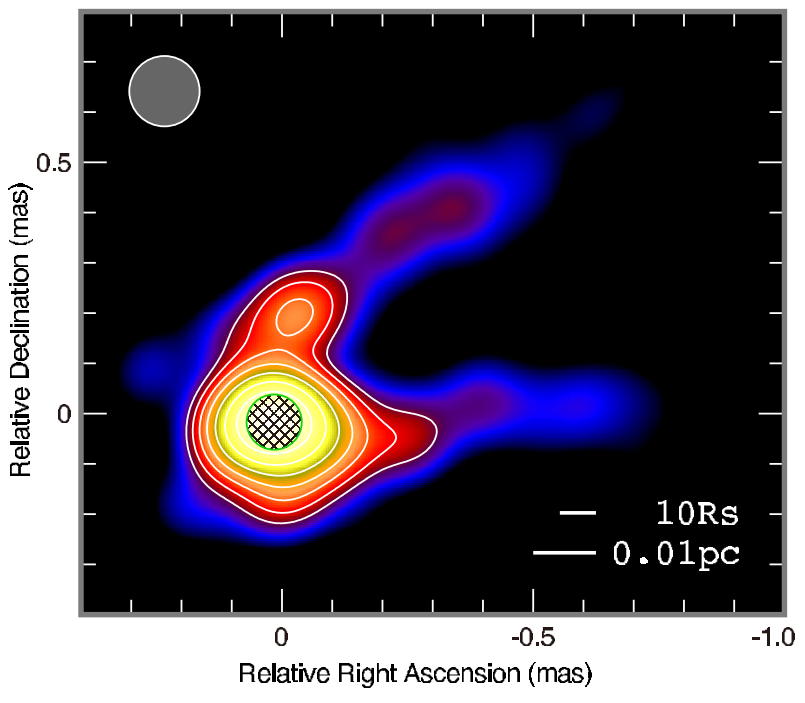

Figure 1. VLBA image of M87 at $43 \mathrm{GHz}$. The the core width (i.e., length perpendicular to the jet axis) derived by the gaussian fitting by AIPS task "JMFIT" is $\theta_{\text {FWHM }}=0.11$ mas (see details in H13). The hatched circle marks the 0.11 mas diameter region explored in this work. Contours start from $3 \sigma$ image rms level and increasing by factors of 1.4 .

$U_{\mathrm{B}} / U_{e}$ at a jet-base than the maximum value obtained in this work (e.g., [23]). For example, the jet flow is heavily Poynting dominated at the jet base with $U_{\mathrm{B}} / U_{e} \sim$ $10^{3}$ in [18]. Therefore, the obtained $U_{e} / U_{\mathrm{B}}$ in this work gives a tight constraint on the initial conditions in relativistic MHD models.

Lastly, we briefly comment on key future works. Observationally, it is crucial to obtain resolved images the radio cores at $43 \mathrm{GHz}$ with space/sub-mm VLBI which would clarify whether there is a sub-structure or not inside $\sim 16$ Rs scale at M87 jet base. Towards this observational final goal, as a first step, it is important to explore physical relations between the results of the present work and observational data at higher frequencies such as $86 \mathrm{GHz}$ and $230 \mathrm{GHz}$ (e.g., [16]; [7]). Indeed, we intend to conduct a new observation of M87 at $86 \mathrm{GHz}$ and we will explore this issue using the new data. Space-VLBI program also could play key role since lower frequency observation can attain higher dynamic range images with a high resolution (e.g., [6]; [22])

\section{Acknowledgment}

We thank A. Tchekhovskoy for useful discussions. This work is partially supported by Grant-in-Aid for Scientific Research, KAKENHI 24540240 (MK) and 24340042 (AD) from Japan Society for the Promotion of Science (JSPS).

\section{References}

[1] Abramowski, A., Acero, F., Aharonian, F., et al. 2012, ApJ, 746, 151

[2] Acciari, V. A., et al. 2009, Science, 325, 444 
[3] Blandford, R. D., \& Znajek, R. L. 1977, MNRAS, 179, 433

[4] Blumenthal, G. R., \& Gould, R. J. 1970, Reviews of Modern Physics, 42, 237 (BG70)

[5] Burbidge, G. R., Jones, T. W., \& Odell, S. L. 1974, ApJ, 193, 43

[6] Dodson, R., Edwards, P. G., \& Hirabayashi, H. 2006, PASJ, 58, 243

[7] Doeleman, S. S., Fish, V. L., Schenck, D. E., et al. 2012, Science, 338, 355

[8] Doi, A., Hada, K., Nagai, H., et al. 2013, this proceedings

[9] Ginzburg, V. L., \& Syrovatskii, S. I. 1965, ARA\&A, 3, 297 (GS65)

[10] Hada, K., Kino, M., Doi, A., et al. 2013, ApJ, in press (H13)

[11] Hada, K., Kino, M., Nagai, H., et al. 2012, ApJ, 760, 52 (H12)

[12] Hada, K., Doi, A., Kino, M., et al. 2011, Nature, 477, 185 (H11)

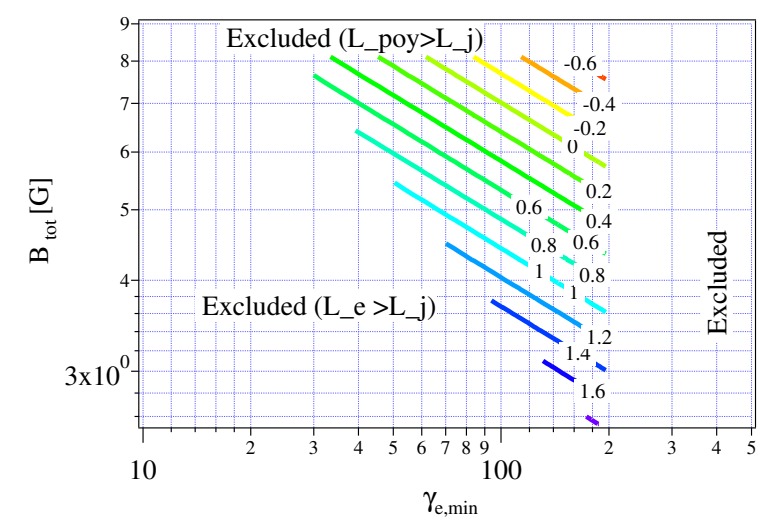

Figure 2. The energy density ratio of $\log \left(U_{e} / U_{\mathrm{B}}\right)$ in the allowed $\gamma_{e \text { min }}-B$ plane with $L_{\mathrm{j}}=3 \times 10^{45} \mathrm{erg} \mathrm{s}^{-1}$ and $p=3.5$.
[13] Kellermann, K. I., \& Pauliny-Toth, I. I. K. 1969, ApJL, 155, L71

[14] Kino, M., Takahara, F., \& Kusunose, M. 2002, ApJ, 564, 97

[15] Kino, M., Takahara, F., Hada, K., \& Doi, A. 2013, in preparation

[16] Krichbaum, T. P., Graham, D. A., Bremer, M., et al. 2006, Journal of Physics Conference Series, 54, 328

[17] Marscher, A. P. 1983, ApJ, 264, 296 (M83)

[18] McKinney, J. C. 2006, MNRAS, 368, 1561

[19] Neronov, A., \& Aharonian, F. A. 2007, ApJ, 671, 85

[20] Owen, F. N., Eilek, J. A., \& Kassim, N. E. 2000, ApJ, 543, 611

[21] Rybicki, G. B., \& Lightman, A. P. 1979, New York, Wiley-Interscience, 1979 (RL79)

[22] Takahashi, R., \& Mineshige, S. 2011, ApJ, 729, 86

[23] Tchekhovskoy, A., Narayan, R., \& McKinney, J. C. 2011, MNRAS, 418, L79 